\title{
Electrophoresis in concentrated dispersions of charged porous spheres
}

\author{
Yan-Ying He, Eric Lee* \\ Department of Chemical Engineering, National Taiwan University, Taipei 10617, Taiwan
}

\section{A R T I C L E I N F O}

\section{Article history:}

Received 27 June 2008

Received in revised form 12 August 2008

Accepted 26 August 2008

Available online 5 September 2008

\section{Keywords:}

Electrophoresis

Porous media

Colloidal phenomenon

Suspension

Polyelectrolyte

Brinkman model

\begin{abstract}
A B S T R A C T
Electrophoresis in a concentrated suspension of charged porous spheres is investigated theoretically, taking into account of the polarization effect of double layer in particular. The double layer thickness and fixed charge density of the porous spheres are arbitrary and double layer overlapping is considered as well. A pseudo-spectral method based on Chebyshev polynomial is used to solve the resulted general electrokinetic equations. Local extrema are observed in the mobility profile as the double layer thickness varies, which is absent in previous theoretical studies neglecting polarization effect but agrees well with the experimental observations in the literature, indicating the importance of polarization effect in correctly interpreting the electrophoretic experimental results. A simple mutual transform of mobility predictions between Levine-Neale and Shilov-Zharkikh boundary conditions is established and confirmed by direct calculations, which facilitates the dialogue between theoretical and experimental researches. In general, the higher the fixed charge density, the more significant the polarization effect is.
\end{abstract}

(c) 2008 Elsevier Ltd. All rights reserved.

\section{Introduction}

Electrophoresis of charged porous spheres under the influence of an externally applied electric field is a very interesting topic, not only in the sense of academic importance but with great potential in practical industrial applications, such as in the waste water treatment, where the waste materials might be modeled as a porous particle. When a colloid immersed in an electrolyte solution is subject to an externally applied electric field, it will set a motion, a phenomenon generally referred to the electrophoresis. Ever since the foundation-laying theoretical analysis made by Von Smoluchowski (1917) who considered the electrophoresis of a single particle with very thin double layer, numerous studies were carried out subsequently to broaden its range of validity one way or another, such as the classic works by Huckel (1924), Henry (1931), Overbeek (1950), Booth (1950) and O'Brien and White (1978), among others. Most of these works are focused on the rigid (hard) spherical colloid, in that no penetration of ions across the colloid surface is allowed. However, there are many colloids which are permeable to ions, as well as the fluid, and form a wide variety of special colloidal suspensions, such as polymer gels or polyelectrolytes. Many biological molecules are polyelectrolytes, such as proteins and DNA. Due to the polymer segments containing functional groups in the polyelectrolyte, it will become charged once immersed in an electrolyte solution.

\footnotetext{
* Corresponding author. Tel./fax: +886223622530.

E-mail address: ericlee@ntu.edu.tw (E. Lee).
}

If the structure of the polyelectrolyte is homogeneous, a constant fixed charge density throughout the resulted porous colloidal structure is generated. To model the fluid flow in a permeable medium, Brinkman (1947) introduced the so-called "Brinkman model", which is essentially a modified Navier-Stokes equation including an extra term representing the corresponding "hydraulic drag force" induced by the presence of the solid part within the porous medium. Although this model is semi-empirical in nature, it fits well with experimental data in general (Happel and Brenner, 1983), and is regarded as the standard approach describing the fluid flow within a porous medium. This idea was also reported independently by Debye and Bueche (1948) nearly at the same time. Appling this model, Hermans (1955) and Hermans and Fujita (1955) considered the electrophoresis of an isolated charged porous sphere immersed in an infinite medium of electrolyte solution, with the constraint that the electric potential possessed by the colloid was low and neglected the polarization effect. The thickness of the electric double layer, the ion cloud surrounding the charged colloid due to the non-even distribution of cations and anions, was finite in their study though. They were able to obtain analytical formulae predicting the electrophoretic mobility of a single porous sphere as a function of double layer thickness. As early as the days when Hermans and Fujita (1955) published their milestone theoretical work, comparison with experimental observations, at least qualitatively, were carried out. Note that the electrophoresis experiment serves as a powerful tool in exploring the surface properties of the colloidal particle in general. Here an experimentalist in biology or biochemistry, for instance, can utilize it to determine the fixed charge density of proteins or DNA. 
Almost the same time, Overbeek and Stigter (1956) considered an identical case and obtained the same mobility solution. They concluded that there are three forces involved in the electrophoresis of charged porous systems: the electric force due to the fixed charge within, the drag force due to the penetration of electrolyte solution into the packed bed type of porous structure, and the fluid flow outside due to the electric body force. Indeed a very insightful elaborations which is applicable in other charged porous sphere systems as well. In general, the mobility was found to increase monotonically in their studies as the double layer gets thicker. Imai and Iwasa (1973) developed a theory of electrophoresis of polyelectrolytes, which considered the relaxation effect, or polarization effect, via the cubic free volume model. They noticed that the applied electric field was strongly depressed near the polyions by the counterions. The case for infinite dilution of the polyion was discussed as a special case. This probably was the only theoretical work published so far addressing the polarization issue in a porous system somehow and somewhat, although the cubic free volume model adopted by them was relatively obscure and the discussion was limited to the infinitely dilute system.

Not many theoretical reports were made since then regarding the charged porous sphere systems until about one decade ago. O'Brien (1995) considered the dynamic mobility of a porous particle subject to an alternating electric field. Formulae for mobility were obtained for various situations when the double layer was very thin. Meanwhile, Keh and his coworkers did a series of studies on the electrokinetic behaviors of charged porous spheres, which included the electric conductivity (1997) and sedimentation velocity and potential (1998) of dilute suspensions, the diffusiophoretic mobility due to an electrolyte gradient (2004), as well as the sedimentation in concentrated suspensions (2006). All of the above analyses were restricted to low electric potential though, hence the polarization effect, the distortion of double layer during motion, was not considered.

Sometimes the colloidal porous sphere is not homogeneous throughout, instead it consists of a hard core sphere with an outer polymer layer of porous structure, the so-called "soft particle" (Ohshima, 1995). Ohshima $(1995,2000,2001)$ conducted a series of theoretical analyses on various electrokinetic behaviors of soft particle systems. Assuming that the polarization effect is negligible (which normally implies low electric potential), as well as a very thin double layer, he was able to obtain approximate expressions for the electrokinetic quantities of interest. The polarization effect on the soft particle systems were later investigated by Lee et al. $(1999,2004)$ for various electrokinetic phenomena. They found that the polarization effect in general tends to reduce the mobility of the particle and exhibits local extrema with varying double layer thickness. Note that as the inner hard core vanishes, the systems reduce to corresponding porous sphere systems. Meanwhile López-García et al. (2003) studied the electrophoretic mobility of a spherical soft particle suspended in a general electrolyte solution numerically using the network method. In particular, Ohshima (2000) obtained simple approximate analytic mobility expressions for concentrated suspensions of charged polyelectrolytes with low electric potential, which can be deduced to the case of Hermans and Fujita (1955). In a following paper the next year, he further showed that the Onsager relation (Ohshima, 2001) between sedimentation potential and electrophoretic mobility still holds in the concentrated suspensions of soft particles.

Traditionally, most studies of the electrophoresis in concentrated suspensions were based on the Levine-Neale boundary condition (Levine and Neale, 1974) for the electrical potential, which was of the Neumann type. However, a new boundary condition (Dirichlet type) was derived according to the Shilov-Zharkikh cell model (Shilov et al., 1981) as suggested by Dukhin et al. (1999), who noticed some inconsistencies in the Levine-Neale model (Levine-Neale and
Shilov-Zharkikh type, LN and SZ, hereafter). The different physical meaning of each condition in the hard sphere model had been discussed by Carrique et al. (2005), who offered a way to conveniently transform the mobility from LN type boundary condition to SZ one, and vice versa. To ascertain which condition has better predictions, Cuquejo et al. (2006) considered high surface potential and polarization effect, compared their electric conductivity results with the experimental data, and found that the predictions by LN model were unable to match the experimental data. Instead, the predictions by SZ model were in full agreement with those experimental results. Their analyses mentioned above, however, confined to hard particle systems only. We find here, as will be elaborated later, a convenient way to transform between the mobility obtained from LN and SZ boundary conditions respectively in concentrated suspensions of charged porous spheres as well.

In summary, we consider here the electrophoresis of a concentrated dispersion of charged porous spheres in an electrolyte solution, with arbitrary electric potential and double layer thickness, hence taking into account of the polarization effect completely. Kuwabara's unit cell model is adopted to describe the system and double layer overlapping is allowed. A pseudo-spectral method (Canuto et al., 1986) based on Chebyshev polynomial is used to solve the resulted general electrokinetic equations numerically. The method is very powerful in solving the general electrokinetic systems of interest (Lee et al., 1999, 2004). It has several desirable properties, such as a fast rate of convergence and the convergent properties are independent of the associated boundary conditions. Also, the mini-max property typically associated with the Chebyshev polynomial is maintained. Key factors are examined for their effects on the electrophoresis, such as the double layer thickness, the fixed charge density of the colloids, the volume fraction of the colloids in the suspension, and so on. In particular, the effect of adopting different boundary conditions on the virtual outer surface in Kuwabara's cell model is examined carefully.

\section{Theory}

Referring to Fig. 1, we consider a concentrated dispersion of spherical porous particles which are penetrable for ions and fluid and carry a fixed charge that may arise from the dissociation of the functional groups. With the incorporation of SZ boundary conditions on the hydrodynamic and electrokinetic aspects, the Kuwabara's unit cell model is adopted, where a dispersion is simulated by a representative cell composed of a porous particle of radius $a$, and an outer concentric shell of electrolyte solution of radius $b$. The spherical coordinates $(r, \theta, \varphi)$ are adopted with their origin located at the center of the particle. An electric field $E_{z}$ is applied externally, which is parallel to $\theta=0$, and $U_{p}$ is the electrophoretic velocity of the particle. The liquid phase contains a $z_{1}: z_{2}$ electrolyte, $z_{1}$ and $z_{2}$ being, respectively, the valence of cations and that of anions. Let $z_{2}=-\alpha z_{1}$ and $H=(a / b)^{3}$, with the latter being the volume fraction of the colloidal dispersion.

The equations governing the present phenomenon comprise those for the electric, concentration, and flow fields respectively, the so-called electrokinetic equations, which are both coupled and highly non-linear set of differential equations. On the basis of Gauss's law, it can be shown that the electric potential $\phi$ is described by the Poisson equation

$$
\begin{aligned}
\nabla^{2} \phi & =-\frac{\rho+\rho_{\mathrm{fix}}}{\varepsilon}, \quad 0<r<a, \\
\nabla^{2} \phi & =-\frac{\rho}{\varepsilon}, \quad a<r<b,
\end{aligned}
$$

where $\rho=\sum_{j} n_{j} z_{j} e$ is the space charge density of mobile ions, $\nabla$ the gradient operator, $e$ the elementary charge, $n_{j}$ and $z_{j}$ the number 


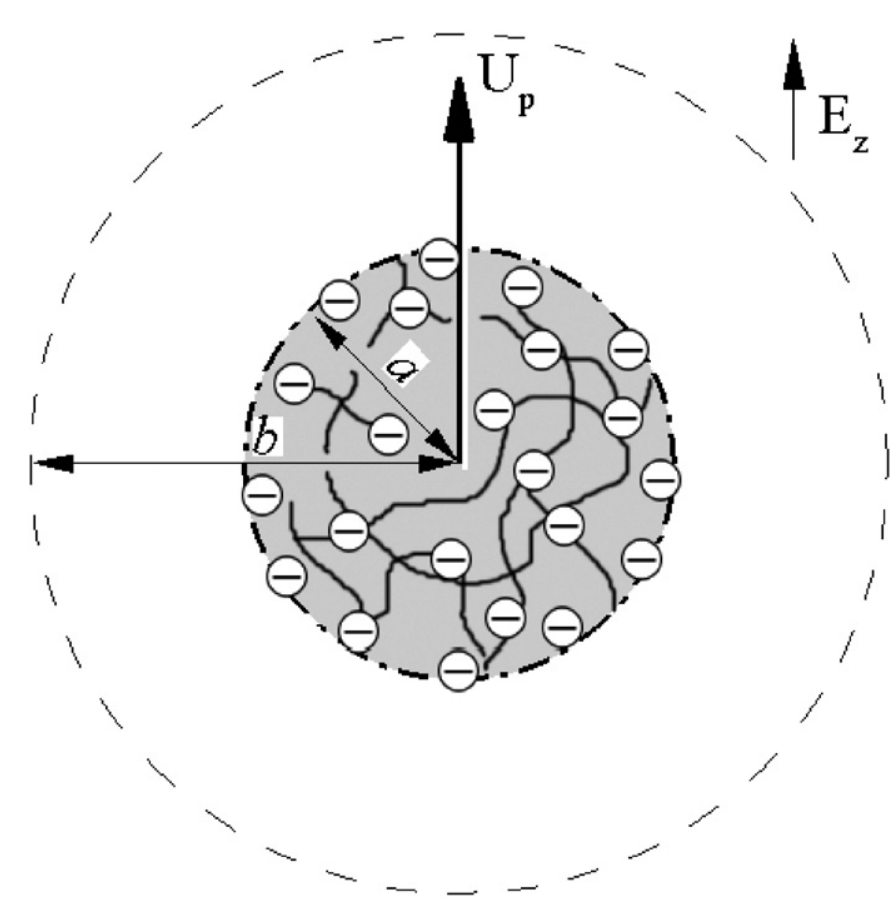

Fig. 1. Configuration of the concentrated suspensions of porous spheres. The radius of particles $a$ and $b$ is the virtual cell boundary, which relates to the crowdness of porous particles. The spherical coordinates are adopted, and the inner gray sphere represents the porous particle characterized by fixed charge density $\rho_{\text {fix }}$ and friction coefficient $\gamma$ (or porosity $\varepsilon_{p}$ ). Small circles represent the fixed charges uniformly distributing inside the porous particle.

concentration and the valence of the ionic species $j$ respectively, and $\varepsilon$ and $\rho_{\text {fix }}$, respectively, the permittivity of the liquid phase and the fixed charge density in the porous particles. We assume that both $\varepsilon$ and $\rho_{\text {fix }}$ are constant. The transport of ions in the ionic solution is governed by the well-known Nernst-Planck equations with convection term

$\mathbf{f}_{j}=-D_{j}\left(\nabla n_{j}+\frac{z_{j} e n_{j}}{k_{B} T} \nabla \phi\right)+n_{j} \mathbf{v}$,

where $\mathbf{f}_{j}$ is the concentration flux of ionic species $j$. The conservation of ionic species yields

$\nabla \cdot \mathbf{f}_{j}=0$

where $D_{j}$ is the diffusivity of the ionic species $j, k_{B}$, and $T$ are, respectively, the Boltzmann constant and the absolute temperature, and $\mathbf{v}$ the liquid velocity relative to the motion of particles. It is assumed that the liquid phase is an incompressible Newtonian fluid with constant physical properties. For the present problem, the flow of liquid is in the creeping-flow region, hence the flow field can be described as follows:

$\nabla \cdot \mathbf{v}=0$

$-\nabla p+\eta \nabla^{2} \mathbf{v}-\rho \nabla \phi-\gamma \mathbf{v}=0, \quad 0<r<a$,

$-\nabla p+\eta \nabla^{2} \mathbf{v}-\rho \nabla \phi=0, \quad a<r<b$,

where $p$ is the pressure, $\eta$ the viscosity of the liquid, and $\gamma$ the frictional coefficient of porous particles. Note that Eq. (5) is the continuity equation, whereas Eq. (6) stands for a modified Stokes equation with an extra term from the frictional force induced in a packed bed type of porous structure. Eq. (6) is often referred to as the Brinkman equation as well. Eq. (7) is the Stokes equation. $\gamma$ can be expressed as $6 \pi \eta a_{S} N_{S}$ when the porous particles are composed of polymers, where $N_{S}$ is the number density and $a_{S}$ the Stokes radius of the polymer segments (Keh and Chen, 2006). Furthermore, $\gamma$ can also be written in the form of $\eta / k_{p}$, where $k_{p}$ is the permeability of the porous particles based on the Darcy's law (Keh and Chang, 2005).

In the case the charged ionic cloud surrounding the particle is only slightly distorted by the application of the electric field, we may replace the original non-linear differential Eqs. (1)-(7) by a set of approximate linear equations by the standard perturbation analysis. Following the treatment of O'Brien and White (1978), the electrical potential $\phi$ is expressed as the sum of the electrical potential in the absence of the applied electric field (or the equilibrium electrical potential), $\phi_{e}$, and that outside a particle arising from the applied electric field, $\delta \phi$; that is, $\phi=\phi_{e}+\delta \phi$. The same procedure can be applied to the concentration of ionic species $j, n_{j}$, the space charge density, $\rho \ldots$ etc. That is, $n_{j}=n_{j e}+\delta n_{j}$ and $\rho=\rho_{e}+\delta \rho$, where $\rho_{e}=\sum_{j} n_{j e} z_{j} e$ and $\delta \rho=\sum_{j} \delta n_{j} z_{j} e$. It should be pointed out that the subscript $e$ represents the equilibrium state and $\delta$ the disturbed state, where the strength of these disturbed properties $\left(\delta \phi, \delta n_{j}\right.$, $\delta \rho \ldots$ ) is proportional to the disturbance, such as the applied electric field and the consequent particle motion. The effect of doublelayer polarization is introduced via $\delta n_{j}$, where $n_{j e}$ and $\delta n_{j}$ can be expressed as

$n_{j e}=n_{j 0} \exp \left(-\frac{z_{j} e}{k_{B} T} \phi_{e}\right)$

$\delta n_{j}=-n_{j e}\left[\frac{z_{j} e}{k_{B} T}\left(\delta \phi+g_{j}\right)\right]$

Here $g_{j}$ represents an equivalent perturbed potential describing the double layer polarization effect and $n_{j 0}$ the bulk number concentration of ionic species $j$. Note that the introduction of $g_{j}$ gives a better mathematical expression on the ion conservation equations.

To simplify the treatment, subsequent discussions are based on scaled symbols, i.e., the governing equations and the associated boundary conditions are rewritten in dimensionless form. The following symbols are chosen for the characteristic variables: the radius of particle, $a$, the thermal electric potential per valence of species 1 , $\phi_{0}$ (defined as $k_{B} T / z_{1} e$ ), the bulk number concentration of the ionic species $1, n_{10}$, and the velocity based on Smoluchowski's theory when an electric field $\phi_{0} / a$ is applied, $U_{E}=\varepsilon \phi_{0}^{2} / \eta a$. Corresponding dimensionless variables are listed as follows: $r^{*}=r / a, n_{j}^{*}=n_{j} / n_{10}$, $\mathbf{E}^{*}=\mathbf{E} /\left(\phi_{0} / a\right), \mathbf{v}^{*}=\mathbf{v} / U_{\mathrm{E}}, \phi^{*}=\phi / \phi_{0}, g_{j}^{*}=g_{j} / \phi_{0}, Q_{\mathrm{fix}}=\rho_{\mathrm{fix}} a^{2} / \varepsilon \phi_{0}$, and $\psi^{*}=\psi / U_{E} a^{2}$. The details of the above derivations can be found elsewhere in our previous publications, such as Lee et al. (2004), among our other previous publications.

\subsection{Dimensionless equilibrium state}

The dimensionless equations for the equilibrium potential is

$$
\begin{aligned}
& \nabla^{* 2} \phi_{e}^{*}=-\frac{(\kappa a)^{2}}{1+\alpha}\left[\exp \left(-\phi_{e}^{*}\right)-\exp \left(\alpha \phi_{e}^{*}\right)\right]-Q_{\mathrm{fix}}, \quad 0<r^{*}<1, \\
& \nabla^{* 2} \phi_{e}^{*}=-\frac{(\kappa a)^{2}}{1+\alpha}\left[\exp \left(-\phi_{e}^{*}\right)-\exp \left(\alpha \phi_{e}^{*}\right)\right], \quad 1<r^{*}<H^{-1 / 3}
\end{aligned}
$$

The equilibrium electric potential $\phi_{e}^{*}$ remains symmetry at the center of the porous particle owing to the spherical symmetry of the equilibrium state. At the particle-liquid interface $(r=a)$, the electrical potential and the electric field are continuous. Besides, the whole cell remains electro-neutrality. Hence the corresponding boundary conditions for the equilibrium potential can be written 
as follows:

$\frac{\mathrm{d} \phi_{e}^{*}}{\mathrm{~d} r^{*}}=0, \quad r^{*}=0$,

$\left.\phi_{e}^{*}\right|_{r^{*}=1^{-}}=\left.\phi_{e}^{*}\right|_{r^{*}=1^{+}},\left.\quad \frac{\mathrm{d} \phi_{e}^{*}}{\mathrm{~d} r^{*}}\right|_{r^{*}=1^{-}}=\left.\frac{\mathrm{d} \phi_{e}^{*}}{\mathrm{~d} r^{*}}\right|_{r^{*}=1^{+}}, \quad r^{*}=1$,

$\frac{\mathrm{d} \phi_{e}^{*}}{\mathrm{~d} r^{*}}=0, \quad r^{*}=H^{-1 / 3}$

\subsection{Dimensionless disturbed state}

Owing to the symmetry nature of the system under consideration, it can be further reduced to a one-dimensional problem. In other words, $\delta \phi^{*}, g_{j}^{*}$, and $\psi^{*}$ can be written as

$\delta \phi^{*}\left(r^{*}, \theta\right)=\Phi\left(r^{*}\right) \cos \theta$,

$g_{j}^{*}\left(r^{*}, \theta\right)=G_{j}^{*}\left(r^{*}\right) \cos \theta$,

$\psi^{*}\left(r^{*}, \theta\right)=\Psi^{*}\left(r^{*}\right) \sin ^{2} \theta$.

Note that in terms of $\psi^{*}$, the dimensionless stream function, the $r$ component of the liquid velocity, $v_{r}^{*}$, and the $\theta$ component of the liquid velocity, $v_{\theta}^{*}$, can be expressed respectively as

$v_{r}^{*}=\frac{-1}{r^{* 2} \sin \theta} \frac{\partial \psi^{*}}{\partial \theta}$,

$v_{\theta}^{*}=\frac{1}{r^{*} \sin \theta} \frac{\partial \psi^{*}}{\partial r^{*}}$.

On the basis of these expressions and the assumption that the applied electric field is weak relative to that induced by the particle itself, it can be shown that the resultant governing equations for $\Phi^{*}, G_{j}^{*}$, and $\Psi^{*}$ become

$$
\begin{gathered}
L^{* 2} \Phi^{*}-\frac{(\kappa a)^{2}}{1+\alpha}\left[\exp \left(-\phi_{e}^{*}\right)+\alpha \exp \left(\alpha \phi_{e}^{*}\right)\right] \Phi^{*} \\
=\frac{(\kappa a)^{2}}{1+\alpha}\left[\exp \left(-\phi_{e}^{*}\right) G_{1}^{*}+\alpha \exp \left(\alpha \phi_{e}^{*}\right) G_{2}^{*}\right],
\end{gathered}
$$

$L^{* 2} G_{1}^{*}-\frac{\mathrm{d} \phi_{e}^{*}}{\mathrm{~d} r^{*}} \frac{\mathrm{d} G_{1}^{*}}{\mathrm{~d} r^{*}}=P e_{1} v_{r}^{*} \frac{\mathrm{d} \phi_{e}^{*}}{\mathrm{~d} r^{*}}$,

$L^{* 2} G_{2}^{*}+\alpha \frac{\mathrm{d} \phi_{e}^{*} \mathrm{~d} G_{2}^{*}}{\mathrm{~d} r^{*}} \frac{\mathrm{d} r^{*}}{\mathrm{~d}}=P e_{2} v_{r}^{*} \frac{\mathrm{d} \phi_{e}^{*}}{\mathrm{~d} r^{*}}$,

$D^{* 4} \Psi^{*}-(\lambda a)^{2} D^{* 2} \Psi^{*}=-\frac{(\kappa a)^{2}}{1+\alpha} \frac{\mathrm{d} \phi_{e}^{*}}{\mathrm{~d} r^{*}}\left[n_{1 e}^{*} G_{1}^{*}+n_{2 e}^{*} G_{2}^{*}\right], \quad 0<r^{*}<1$,

$D^{* 4} \Psi^{*}=-\frac{(\kappa a)^{2}}{1+\alpha} \frac{\mathrm{d} \phi_{e}^{*}}{\mathrm{~d} r^{*}}\left[n_{1 e}^{*} G_{1}^{*}+n_{2 e}^{*} G_{2}^{*}\right], \quad 1<r^{*}<H^{-1 / 3}$.

In these expressions, $\kappa^{-1}=\left[\varepsilon k_{B} T / \sum_{j} z_{j}^{2} e^{2} n_{j 0}\right]^{1 / 2}$ is the Debye screening length, $P e_{j}=U_{E} a / D_{j}$, is the Peclet number of ionic species $j$. Moreover, $\lambda^{-1}$ defined as $(\eta / \gamma)^{1 / 2}$ or $k_{p}{ }^{1 / 2}$ is the Debye-Bueche screening length of the porous particles. The linear operators $L^{* 2}$ and $D^{* 4}$ are defined, respectively, by

$L^{* 2}=\frac{\mathrm{d}^{2}}{\mathrm{~d} r^{* 2}}+\frac{2}{r^{*}} \frac{\mathrm{d}}{\mathrm{d} r^{*}}-\frac{2}{r^{* 2}}$,

$D^{* 4}=D^{* 2} D^{* 2}=\left(\frac{\mathrm{d}^{2}}{\mathrm{~d} r^{* 2}}-\frac{2}{r^{* 2}}\right)^{2}$.

Eqs. (27)-(31) shown below are the corresponding boundary conditions for $\delta \phi, g_{j}$, and $\psi$ in terms of $\Phi^{*}, G_{j}^{*}$, and $\Psi^{*}$. At the center of the particle, $\mathbf{v}$ is finite, and $\delta \phi$ and $g_{j}$ are set to zero as the reference point of potential. The dimensionless boundary conditions thus are

$\Phi^{*}=G_{j}^{*}=\Psi^{*}=\frac{\mathrm{d} \Psi^{*}}{\mathrm{~d} r^{*}}=0, \quad r^{*}=0$.

At the particle-liquid interface, both $\delta \phi$ and $\nabla \delta \phi$ are continuous, due to the assumption that the relative permittivity $\varepsilon_{r}$ takes the same value both inside and outside the porous particles. The boundary conditions for $g_{j}$ are described in Eq. (29), which comes from the continuity of ion concentration and flux. Also, the velocity of fluid and its normal and tangential stresses are assumed continuous. Eqs. (28)-(30) reflect the above physical deductions.

$$
\begin{aligned}
& \left.\Phi^{*}\right|_{r^{*}=1^{-}}=\left.\Phi^{*}\right|_{r^{*}=1^{+}},\left.\quad \frac{\mathrm{d} \Phi^{*}}{\mathrm{~d} r^{*}}\right|_{r^{*}=1^{-}}=\left.\frac{\mathrm{d} \Phi^{*}}{\mathrm{~d} r^{*}}\right|_{r^{*}=1^{+}}, \quad r^{*}=1, \\
& \left.G_{j}^{*}\right|_{r^{*}=1^{-}}=\left.G_{j}^{*}\right|_{r^{*}=1^{+}},\left.\quad \frac{\mathrm{d} G_{j}^{*}}{\mathrm{~d} r^{*}}\right|_{r^{*}=1^{-}}=\left.\frac{\mathrm{d} G_{j}^{*}}{\mathrm{~d} r^{*}}\right|_{r^{*}=1^{+}}, \quad r^{*}=1, \\
& \left.\Psi^{*}\right|_{r^{*}=1^{-}}=\left.\Psi^{*}\right|_{r^{*}=1^{+}},\left.\quad \frac{\mathrm{d} \Psi^{*}}{\mathrm{~d} r^{*}}\right|_{r^{*}=1^{-}}=\left.\frac{\mathrm{d} \Psi^{*}}{\mathrm{~d} r^{*}}\right|_{r^{*}=1^{+}}, \\
& \left.\frac{\mathrm{d}^{2} \Psi^{*}}{\mathrm{~d} r^{* 2}}\right|_{r^{*}=1^{-}}=\left.\frac{\mathrm{d}^{2} \Psi^{*}}{\mathrm{~d} r^{* 2}}\right|_{r^{*}=1^{+}}, \quad \mathrm{d}^{3} \Psi^{*} \\
& {\left.\left[\frac{\mathrm{d}^{3} r^{* 3}}{\mathrm{~d}}-(\lambda a)^{2} \frac{\mathrm{d} \Psi^{*}}{\mathrm{~d} r^{*}}\right]\right|_{r^{*}=1^{-}}=\left.\frac{\mathrm{d}^{*}}{\mathrm{~d} r^{* 3}}\right|_{r^{*}=1^{+}}, \quad r^{*}=1 .}
\end{aligned}
$$

At the outer boundary of the cell (virtual surface), SZ boundary condition mentioned earlier is applied, which states that the concentration of ionic species reaches equilibrium value on the cell surface. Furthermore, according to the analysis of Kuwabara (1959), no fluid transport between each cell and the vorticity vanishes at the outer boundary of a cell. Therefore,

$\Phi^{*}=-H^{-1 / 3} E_{z}^{*}, \quad G_{j}^{*}=-\Phi^{*}$,
$\Psi^{*}=\frac{1}{2} r^{* 2} U_{p}^{*}, \quad D^{* 2} \Psi^{*}=0, \quad r^{*}=H^{-1 / 3}$.

According to O'Brien and White (1978), the present problem can be divided into two sub-problems. In the first problem, the particle moves in the absence of the applied electric field, $E_{z}$, and in the second problem $E_{z}$ is applied, but the particle is kept fixed instead. In the first problem the total force acting on a particle in the vertical direction, $F_{1}$, is proportional to its electrophoretic velocity, $U_{p}$, while in the second problem the total force, $F_{2}$, is proportional to the applied electric field, $E_{z}$. Therefore, we have $F_{1}=c_{1} U_{p}^{*}$ and $F_{2}=c_{2} E_{z}^{*}$, where $c_{1}$ is independent of $U_{p}^{*}$, and $c_{2}$ is independent of $E_{z}^{*}$. The dimensionless electrophoretic mobility can be defined as $\mu_{m}^{*}=U_{p}^{*} / E_{z}^{*}$. Because $F_{1}+F_{2}=0$ at steady state, $\mu_{m}^{*}$ can be expressed as

$\mu_{m}^{*}=\frac{U_{p}^{*}}{E_{z}^{*}}=-\frac{c_{2}}{c_{1}}$.

Note that both $c_{1}$ and $c_{2}$ are independent of $U_{p}^{*}$ and $E_{z}^{*}$. The forces acting on a particle $F_{i}$ comprises the electric force $F_{i e}$ and the hydrodynamic force $F_{i d}$, where the subscript $i$ represents subproblem 1 and 2 . The electric and hydrodynamic forces acting on a particle can be evaluated, respectively, by

$F_{i e}=\iiint_{r \leqslant a} \rho_{\text {fix }}(-\nabla \phi) \mathrm{d} V \cdot \mathbf{i}_{z}=-\left.\frac{4}{3} \pi \varepsilon \phi_{0}^{2} Q_{\mathrm{fix}} \Phi^{*}\right|_{r^{*}=1}$,

$F_{i d}=\iiint_{r \leqslant a} \gamma \mathbf{v} \mathrm{d} V \cdot \mathbf{i}_{z}=-\left.\frac{8}{3} \pi \varepsilon \phi_{0}^{2}(\lambda a)^{2} \Psi^{*}\right|_{r^{*}=1}$,

where $\mathbf{i}_{z}$ is the unit vector in the $z$ direction. Once $F_{i e}$ and $F_{i d}$ are evaluated, $c_{i}$ and $\mu_{m}^{*}$ can be obtained easily. 


\section{Results and discussion}

Since there is uniform distribution of fixed charge within the porous sphere, co-ions will be expelled into the outer solution phase while the counter-ions will be attracted inward, result in a nonuniform distribution of ions at equilibrium both inside and outside of the porous spheres. Hence the traditional double layer notion in systems of rigid hard colloidal spheres has to be modified somehow to reflect this fact that diffuse double layer of porous sphere occurs across the particle-solution interface. Moreover, the traditional concept of zeta potential at the shear plane is no longer applicable here. Instead, the uniform fixed charged density is much more suitable to represent the overall electric situation of the porous colloid under consideration. Its dimensionless form is defined as follows:

$Q_{\mathrm{fix}}=\frac{\rho_{\mathrm{fix}} a^{2}}{\varepsilon \phi_{0}}$.

To facilitate the subsequent analysis, we define a scaled mobility as follows accordingly:

$\mu_{s}^{*}=\frac{\mu_{m}^{*}}{Q_{\mathrm{fix}} /(\lambda a)^{2}}$,

when the double layer is considered infinitely thin, this scaled mobility, $\mu_{s}^{*}$, will become unity, which means the dimensionless mobility equals to $Q_{\text {fix }} /(\lambda a)^{2}$, the electrophoretic mobility of an individual polymer segment or monomer, according to the study of Fujita (1957).

In a subsequent note by Fujita (1957), they referred to an experimental work by Nagasawa et al. (1958), indicating that electrophoretic mobility should exhibit a local extremum with varying $\kappa a$, the dimensionless reciprocal of double layer thickness. In recent years, Garcíya-Salinas et al. (2001) and Chen and Tallarek (2003) conducted experiments on the electrophoresis of porous particles and both found local extrema in their results, which are consistent with the typical predictions in theoretical analyses incorporating polarization effect, whereas absent in corresponding theoretical approaches assuming low electric potential hence neglecting polarization effect.
The experimental results mentioned above suggest a great need to explore the charged porous spheres further with polarization effect considered, from the practical point of view.

As mentioned earlier in the introduction section, Hermans and Fujita (1955) studied the electrophoresis phenomenon of a single porous sphere with low zeta potential and provided an analytical formulae for the calculation of the electrophoretic mobility. This is by far the most important bench-mark testing case available in the literature for comparison purpose, as Ohshima (2000) did to test his result for the situation of infinitely thin double layer. We compare our calculation results with those of Hermans and Fujita (1955) as well by setting the fixed charge density $Q_{\mathrm{fix}}$ to be low enough in our calculations, which correspond to their assumption that the double layer remains spherically symmetric in the presence of the applied electric field, i.e., taking no account of double layer polarization, a deduction consistent with the Debye-Huckel approximation employed by them. As the volume fraction $H$ is close to zero, our system considered above tends to a very dilute suspension, and approaches the one considered by Hermans and Fujita (1955).

\subsection{Effect of double layer thickness}

Corresponding calculation results are shown in Figs. 2(a) and (b) where the electrophoretic mobility of the porous colloidal particles is depicted as a function of scaled double layer thickness $\kappa a$ at various volume fractions. We observe that the electrophoretic mobility of porous particles is still a positive value as $\kappa a$ tends to zero in both Figs. 2(a) and (b), whereas in the corresponding case of rigid spheres with constant zeta potential (Lee et al., 1999) is zero. A very small $\kappa a$ indicates very thick double layer, hence nearly perfect double layer mixing, which nullifies electric driving force in suspensions of hand spheres, as reported in the literature by various groups. However, the fixed charges within the porous particles, which is absent in the rigid particle situation, still provide an extra electric driving force, hence results in a positive asymptotically constant mobility. On the other hand, as the double layer gets very thin, the majority of the counterions will be confined to the interior of the porous sphere, hence alleviates the strength of the electric driving force by a

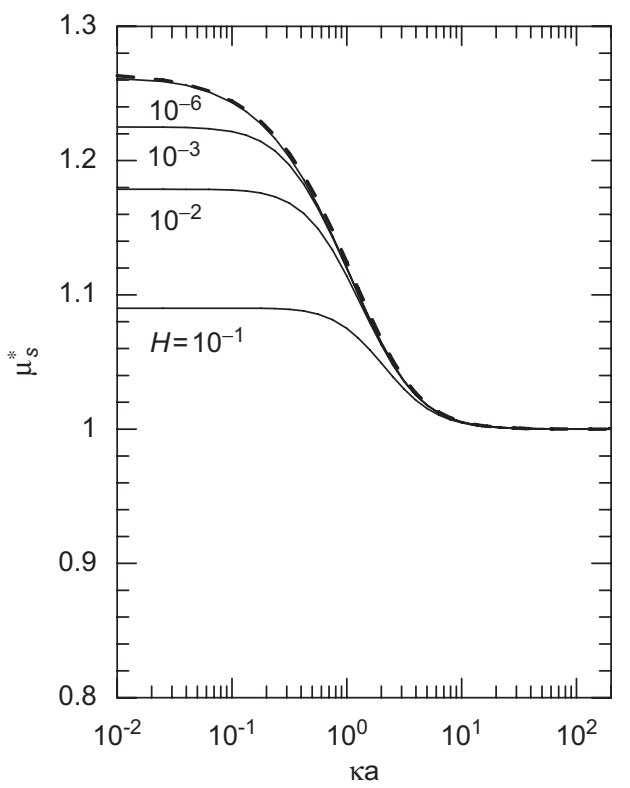

b

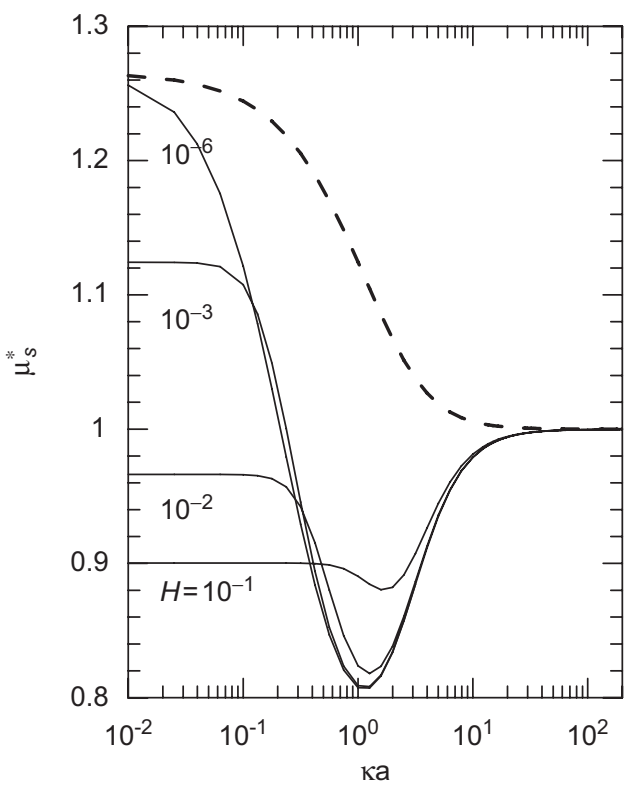

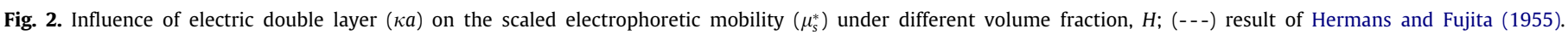
Key: $\lambda a=1, P e_{j}=0.1$. (a) $Q_{\mathrm{fix}}=1$; (b) $Q_{\mathrm{fix}}=10$. 
a

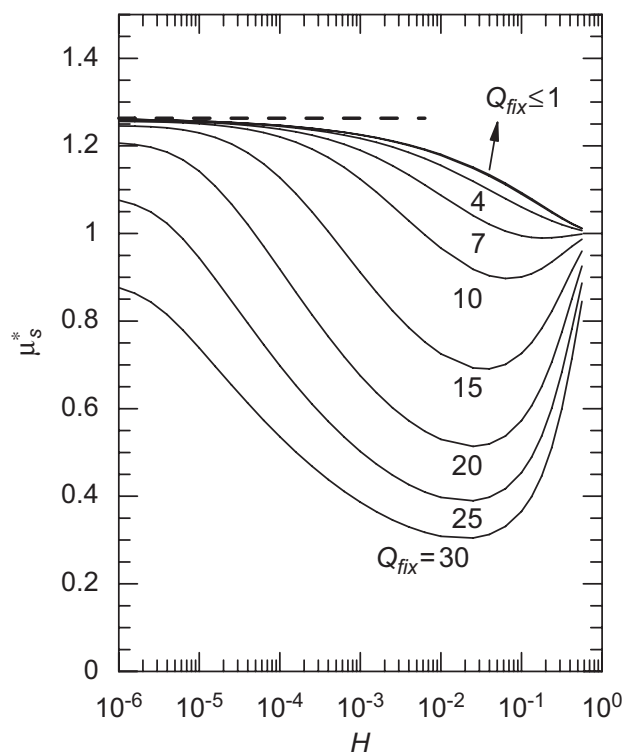

b

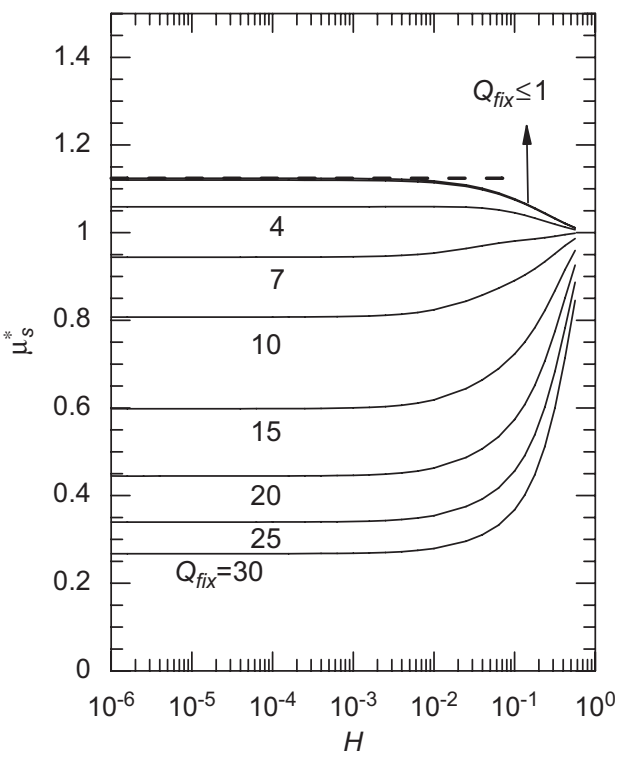

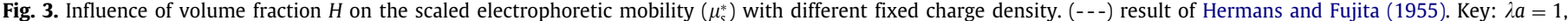
$P e_{j}=0.1$. (a) $\kappa a=0.01$; (b) $\kappa a=1$.

the fixed charge, and leads to a monotonic decrease of electrophoretic mobility.

As shown in Fig. 2(b), however, the behavior of scaled electrophoretic mobility is qualitatively different from that in Fig. 2(a) except when double layer is very thin. This is mainly due to the higher electric potential involved, or, to be exact, the double layer polarization, which cannot be ignored if the charge density $Q_{\mathrm{fix}}$ is high. In addition, the effect of double layer overlapping, a phenomenon typical in concentrated suspension, tends to decrease the electrophoretic mobility as well (Lee et al., 1999) when double layer gets thicker and volume fraction becomes higher. The effect of double layer polarization and the effect of double layer overlapping will be further examined again later.

\subsection{Effect of double layer polarization}

To shed more light on the effect of double layer polarization, we further analyze the role of the charge density $Q_{\mathrm{fix}}$ in Figs. 2(a) and (b). The fixed charges in porous particles electrostatically repulse co-ions flowing into the particles and attract counterions instead at the same time. This inflow of counterions depresses the electric field near the particle surface to some degree, the so-called shielding effect (Imai and Iwasa, 1973). Moreover, an induced opposite electric field is generated when the particle is in motion, and consequently reduces the electrophoretic mobility, the so-called polarization effect. In Fig. 2(a), where $Q_{\text {fix }}$ is low, the general behavior is qualitatively similar to the Hermans and Fujita's (1955) case except that the mobility now is somewhat lower due to the hindrance effect of double layer overlapping. The deviation is not serious since the polarization effect is relatively obscure when the double layer is thin or the $Q_{\mathrm{fix}}$ is low.

When the $Q_{\mathrm{fix}}$ is high, however, the polarization effect becomes so dominant at some range of $\kappa a$ that local extremes appear in the mobility profile, as shown in Fig. 2(b). The reason for the initial decrease of mobility as $\kappa a$ increases around $\kappa a=1$ is due to, as just mentioned, the polarization effect. As $\kappa a$ increases further, the mobility rises since the shielding effect suppressing the double layer gradually dies out. If $\kappa a$ increases to infinity, the mobility eventually reduces to unity, regardless of the variation of particle volume fraction. Overall, the double layer polarization has a great impact on the electrophoretic mobility of porous particles except when the double layer is very thin. The higher the $Q_{\mathrm{fix}}$, the more significant the polarization effect, and a drastic local extremum is observed in mobility profile.

\subsection{Influence of volume fraction}

Our results shown in Fig. 3 extended the predictions by Hermans and Fujita (1955) to cover the range of concentrated suspensions, and depicted the range of error in applying their analytical formulae, where $H$ represents the volume fraction of porous particles. The deviation from the dilute assumption gets more severe as the volume fraction of colloids gets higher, due to the hindrance effect from the hydrodynamic interactions between the neighboring particles. Note that although in principle it is possible to have near-unity volume fraction in the cell model, the maximum possible volume fraction allowed in practice is about 0.74 with the densest packing arrangement of spherical particles.

As $H$ decreases to approximately 0.1 in Figs. 3(a) and (b), the mobility decreases except when the $Q_{\mathrm{fix}}$ is low enough to ignore the double layer polarization. In this case, the amount of ions dissociated from particles is far greater than that of added salts, which makes solution behave like a salt-free system. Hence, double layer thickness has no influence on the scaled mobility.

As $H$ decreases further, discrepancy in behaviors appears in mobility profiles. While most of mobility profiles in Fig. 3(b) remains a lower horizontal behavior, all of the profiles in Fig. 3(a) start to climb up due to the double layer overlapping effect-the mobility profile rises due to less double layer overlapping and then gradually becomes horizontal as double layer shrinks within the virtual cell eventually. In Fig. 3(b), however, no such decrease of double layer overlapping effect takes place even if $H$ approaches unity. This is because the double layer will never touch the virtual cell boundary when double layer thickness, $\kappa^{-1}$, is equal to the particle radius, $a$.

\subsection{Effect of dimensionless parameter, $Q_{\mathrm{fix}} /(\lambda a) 2$, on the scaled mobility}

The ratio $Q_{\mathrm{fix}} /(\lambda a)^{2}$, as mentioned earlier, represents the dimensionless electrophoretic mobility as the double layer is infinitely thin, 
a

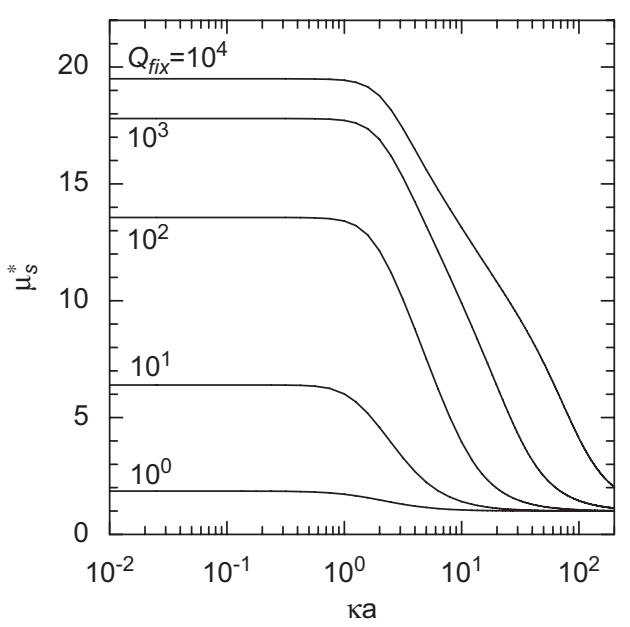

b

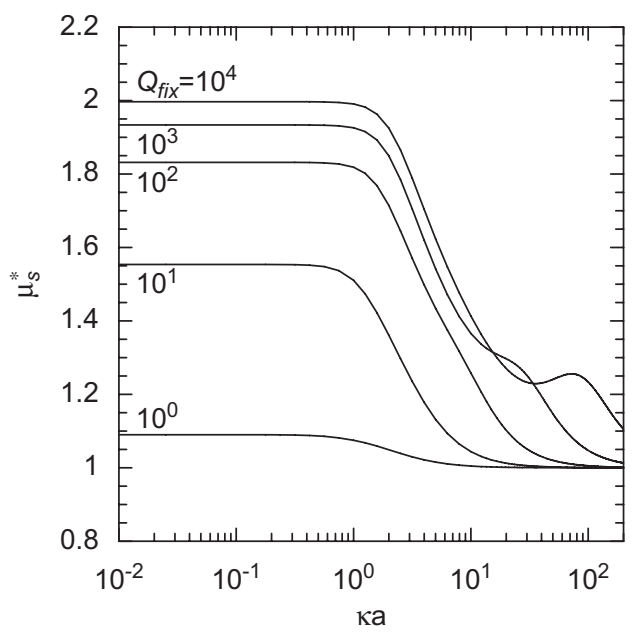

C

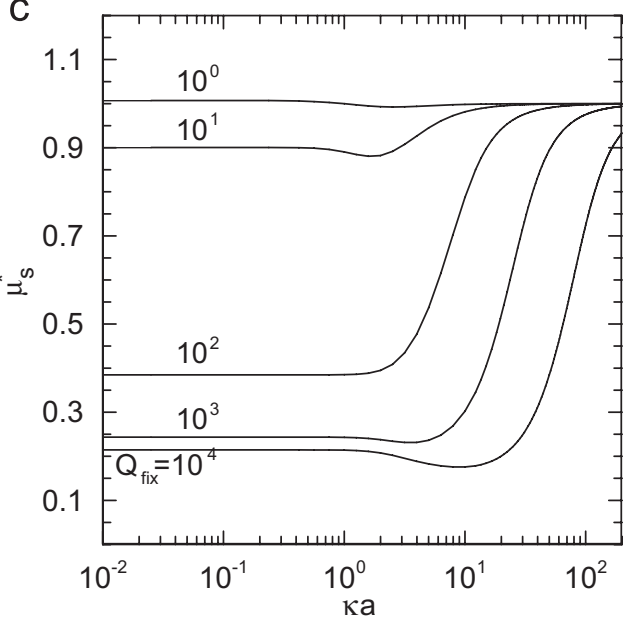

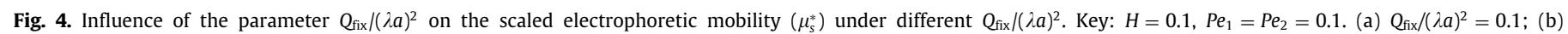
$Q_{\mathrm{fix}} /(\lambda a)^{2}=1 ;(\mathrm{c}) Q_{\mathrm{fix}} /(\lambda a)^{2}=10$.

or that of an individual polymer segment or monomer that compose the porous particles (Fujita, 1957). Here we observe that the ratio $Q_{\text {fix }} /(\lambda a)^{2}$ greatly influences the mobility profile of porous particles. It actually serves as a convenient dimensionless parameter to characterize the charge property of the porous particles in practical applications.

Fig. 4 reveals the mobility as a function of $\kappa a$ at various $Q_{\mathrm{fix}} /(\lambda a)^{2}$. In Fig. $4(a)$, where the ratio $Q_{\mathrm{fix}} /(\lambda a)^{2}$ is low, hydrodynamic drag force dominates the mobility profiles which decline with increasing $\kappa a$. In contrast, when the ratio $Q_{\text {fix }} /(\lambda a)^{2}$ is very high as illustrated in Fig. 4(c), electric driving force decreases greatly due to the polarization effect, hence the mobility rises generally with increasing $\kappa a$. Local extrema appear owing to the competition between forces due to the hydrodynamic flow and the polarization effect. The ratio $Q_{\text {fix }} /(\lambda a)^{2}$ turns out to be the most important parameter on deciding the mobility profile.

\subsection{Effect of cell model boundary conditions}

The difference between LN and SZ boundary conditions is from the definition of "externally applied electric field". The mobility predictions based on LN and SZ boundary conditions respectively are different when double layer overlapping occurs. Carrique et al. (2005) showed recently that the two sets of mobility data are related via the following equations:

$\mu_{S, L N}^{*}=\left.\frac{\Phi^{*} / r^{*}}{\mathrm{~d} \Phi^{*} / \mathrm{d} r^{*}}\right|_{r^{*}=H^{-1 / 3}} \cdot \mu_{s, S Z}^{*}$

As shown graphically in Figs. 5(a) and (b), by direct numerical calculations, the above relation holds here as well for the charged porous spheres.

\subsection{Comparison with experimental results}

An experimental study of electrophoresis was available in early days by Nagasawa et al. (1958). The particular suspension used there was polyvinyl sulfate in sodium chloride solutions (Na-PVS in $\left.\mathrm{NaCl}_{(\text {aq. }}\right)$. Examining the mobility profile as a function of varying concentration of $\mathrm{NaCl}$, or $\kappa a$ in proportion, they found a local maximum at low concentration (Fig. 8 in their paper). This did not agree with the theoretical prediction by Hermans and Fujita's theory, which yielded a profile asymptotic to infinity at low concentration. However this experimental observation agreed perfectly well with our current theory which takes into account the polarization effect.

Moreover, in a recent paper by Garcíya-Salinas et al. (2001) where the electrophoretic behavior of a polyelectrolyte, poly $(\mathrm{N}-$ isopropylacrylamide) microgel, was investigated experimentally. The electrophoretic mobility profile presented there as a function 
a

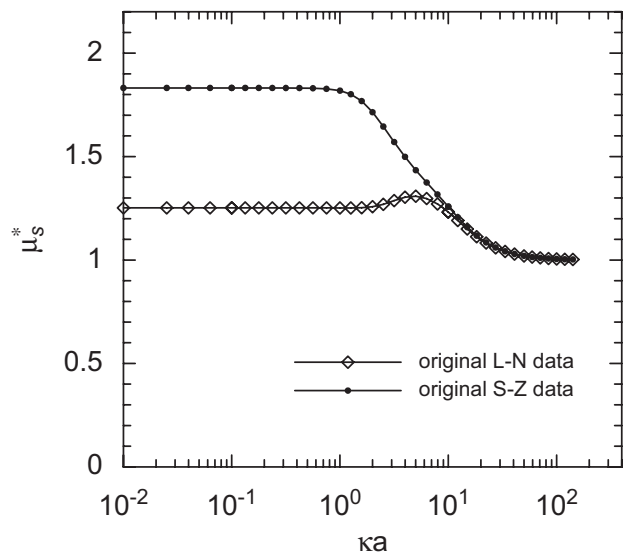

b

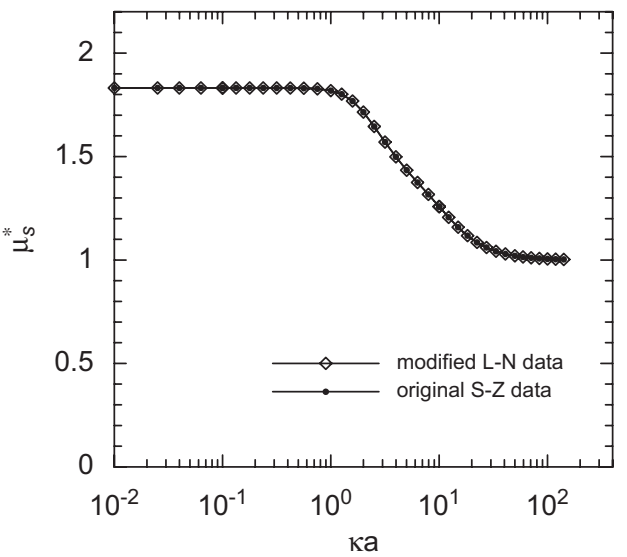

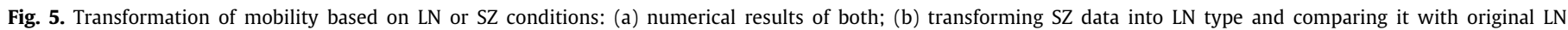
data. Key: $H=0.1, Q_{\text {fix }}=100, Q_{\text {fix }} /(\lambda a)^{2}=1, P e_{1}=P e_{2}=0.1$.

of electrolyte concentration (Fig. 5 in their paper) fits excellently well with our results such as the curve $Q_{\text {fix }}=10^{3}$ in Fig. 4(b), as far as the local characteristic shoulder-like shape is concerned, which again cannot be predicted by earlier theoretical approaches neglecting double layer polarization.

\section{Conclusion}

Electrophoresis in a concentrated suspension of charged porous spheres is investigated theoretically here on the basis of Kuwabara's cell model. The fixed charge density of the colloid can be arbitrary thus the polarization effect is taken into account, a major step further compared to previous studies confined to low fixed charge density. A pseudo-spectral method based on Chebyshev polynomial is used to solve the resulted general electrokinetic equations, and various key parameters were examined on their effects on mobility. We find:

(1) As double layer becomes thinner, the scaled mobility either decreases or increases depending on the competition outcome between the forces due to hydrodynamic flow inside porous particles and the polarization effect, and eventually tends to unity as the double layer becomes infinitely thin, regardless of volume fraction of the colloids.

(2) When the suspension gets more and more concentrated, the scaled mobility may decrease owing to double layer overlapping effect and then approaches to unity eventually.

(3) Double layer polarization has an effect to decrease the mobility in general, and local extrema are observed.

(4) The simple transform relations between Levine-Neale and Shilov-Zharkikh boundary conditions in hard colloids holds as well here for porous spheres.

Overall, polarization effect is crucial in accurately predicting the mobility as the fixed charge density is high, consistent with experimental observations available in literature.

\section{References}

Booth, F., 1950. The cataphoresis of spherical, solid non-conducting particles in a symmetrical electrolyte. Proceedings of the Royal Society of London Series A-Mathematical and Physical Sciences 203, 514-533.

Brinkman, H.C., 1947. A calculation of the viscous force exerted by a flowing fluid on a dense swarm of particles. Applied Scientific Research Section A-Mechanics Heat Chemical Engineering Mathematical Methods 1, 27-34.

Canuto, C., Hussaini, M.Y., Quarteroni, A., Zang, T.A., 1986. Spectral Methods in Fluid Dynamics. Springer, New York.
Carrique, F., Cuquejo, J., Arroyo, F.J., et al., 2005. Influence of cell-model boundary conditions on the conductivity and electrophoretic mobility of concentrated suspensions. Advances in Colloid and Interface Science 118, 43-50.

Chen, G., Tallarek, U., 2003. Effect of intraparticle porosity and double layer overlap on electrokinetic mobility in multiparticle systems. Langmuir 19, 10901-10908.

Cuquejo, J., Jimenez, M.L., Delgado, A.V., et al., 2006. Numerical and analytical studies of the electrical conductivity of a concentrated colloidal suspension. Journal of Physical Chemistry B 110, 6179-6189.

Debye, P., Bueche, A.M., 1948. Intrinsic viscosity, diffusion, and sedimentation rate of polymers in solution. Journal of Chemical Physics 16, 573-579.

Dukhin, A.S., Shilov, V., Borkovskaya, Y., 1999. Dynamic electrophoretic mobility in concentrated dispersed systems. Cell model. Langmuir 15, 3452-3457.

Fujita, H., 1957. Notes on the calculation of electrophoresis of polyelectrolytes with partial free-drainage. Journal of the Physical Society of Japan 12 (8), 968-973.

Garcíya-Salinas, M.J., Romero-Cano, M.S., de las Nieves, F.J., 2001. Electrokinetic characterization of poly(n-isopropylacrylamide) microgel particles: effect of electrolyte concentration and temperature. Journal of Colloid and Interface Science 241, 280-285.

Happel, J., Brenner, H., 1983. Low Reynolds Number Hydrodynamics: With Special Application to Particulate Media. Nijhoff, New York pp. 390-391.

Henry, D.C., 1931. The cataphoresis of suspended particles part I-the equation of cataphoresis. Proceedings of the Royal Society of London Series A-Containing Papers of a Mathematical and Physical Character 133, 106-129.

Hermans, J.J., 1955. Sedimentation and electrophoresis of porous spheres. Journal of Polymer Science 18, 527-534.

Hermans, J.J., Fujita, H., 1955. Electrophoresis of charged polymer molecules with partial free drainage. Koninklijke Nederlandse Akademie Wetenschappen Proceedings, Series B 58, 182.

Huckel, E., 1924. The cataphoresis of the sphere. Physikalische Zeitschrift 25, 204-210.

Imai, N., Iwasa, K., 1973. Theory of electrophoresis of polyelectrolytes. Israel Journal of Chemistry 11 (2-3), 223-233.

Keh, H.J., Chang, Y.C., 2005. Creeping motion of an assemblage of composite spheres relative to a fluid. Colloid and Polymer Science 283, 627-635.

Keh, H.J., Chen, W.C., 2006. Sedimentation velocity and potential in concentrated suspensions of charged porous spheres. Journal of Colloid and Interface Science 296, 710-720.

Kuwabara, S., 1959. The forces experienced by randomly distributed parallel circular cylinders or spheres in a viscous flow at small Reynolds numbers. Journal of the Physical Society of Japan 14 (4), 527-532.

Lee, E., Chu, J.W., Hsu, J.P., 1999. Electrophoretic mobility of a concentrated suspension of spherical particles. Journal of Colloid and Interface Science 209, 240-246.

Lee, E., Chou, K.T., Hsu, J.P., 2004. Electrophoresis of a concentrated dispersion of spherical particles covered by an ion-penetrable membrane layer. Journal of Colloid and Interface Science 280, 518-526.

Levine, S., Neale, G.H., 1974. The prediction of electrokinetic phenomena within multiparticle systems: 1 . Electrophoresis and electroosmosis. Journal of Colloid and Interface Science 47 (2), 520-529.

López-García, J.J., Grosse, C., Horno, J., 2003. Numerical study of colloidal suspensions of soft spherical particles using the network method: 1 . DC electrophoretic mobility. Journal of Colloid and Interface Science 265, 327-340.

Nagasawa, M., Soda, A., Kagawa, I., 1958. Electrophoresis of Polyelectrolyte in Salt Solutions. Journal of Polymer Science XXXI, 439-451.

O'Brien, R.W., 1995. The dynamic mobility of a porous particle. Journal of Colloid and Interface Science 171, 495-504.

O'Brien, R.W., White, L.R., 1978. Electrophoretic mobility of a spherical colloidal particle. Journal of the Chemical Society-Faraday Transactions II 74, 1026-1607. 
Ohshima, H., 1995. Electrophoresis of soft particles. Advances in Colloid and Interface Science 62, 189-235.

Ohshima, H., 2000. Electrophoretic mobility of soft particles in concentrated suspensions. Journal of Colloid and Interface Science 225, 233-242.

Ohshima, H., 2001. Electrokinetic phenomena in a concentrated suspension of soft particles. Colloids and Surface A: Physicochemical and Engineering Aspects 195, 129-134.

Overbeek, J.Th.G., 1950. Quantitative interpretation of the electrophoretic velocity of colloids. Advanced in Colloid Science 3, 97-135.
Overbeek, J.Th.G., Stigter, D., 1956. Electrophoresis of polyelectrolytes with partial drainage. Journal of the Royal Netherlands Chemical Society 75 (4), 543-554.

Shilov, V.N., Zharkikh, N.I., Borkovskaya, Y.B., 1981. Theory of non-equilibrium electrosurface phenomena in concentrated disperse systems. 1. Application of non-equilibrium thermodynamics to cell model of concentrated dispersions. Colloid Journal of the USSR 43 (3), 434-438.

Von Smoluchowski, M., 1917. Experiments on a mathematical theory of kinetic coagulation of colloid solutions. Zeitschrift fur Physikalische ChemieStochiometrie und Verwandtschaftslehre 92 (2), 129-168. 